\title{
Human Activity Recognition using Active Learning Methodology
}

\author{
K.R.Baskaran, M.N.Saroja
}

\begin{abstract}
In current technology, presenting detailed and exact information about one's daily activities is the major task in artificial intelligence. This paper represents the multiple classification techniques used to monitor the behaviours of aging people. It can also play an important role in health care monitoring system and surveillance systems. Human Activity Recognition (HAR) dataset is used for evaluating and comparing the prediction accuracy of the dictionary learning algorithm, Naive Bayes and $\mathrm{J48}$ algorithms. Based on the classification, J48 algorithm is superior compared to other classifier algorithms. J48 and Naïve Bayes machine learning algorithms are evaluated on WEKA tool and their efficiency is compared with Dictionary learning algorithm for achieving better results on the given dataset.
\end{abstract}

Index Terms-Machine learning, HAR, Dictionary learning, ADL problem.

\section{INTRODUCTION}

In recent times, the presence of old aged people alone at home is an important issue considering their safety and security. Monitoring the day-to-day activities of elderly people is an important challenge in current technology. Smart Gadgets are being developed to monitor their activities. In healthcare, old aged homes and self-assisted centre monitoring the activities such as falls and their gestures are major issues in competitive technologies. Fall detection systems are also prevalent. Nevertheless, accuracy and efficiency is an important point to be considered. It is evaluated based on the machine learning algorithms used by the Smart System.

For comparing the efficiency of machine learning algorithms in predicting the daily living activities, Human Activity Recognition (HAR) dataset is being used. Machine learning algorithms are applied to the HAR dataset and the classifier accuracy and efficiency of prediction are being compared. The problems observed in the existing classifier model as follows: developing classifier modules are highly expensive, reliability is not guaranteed, interpretability of the system not ascertained.

In addition to the available algorithms, Dictionary learning algorithm is also used for predicting its performance and accuracy.

\section{LITERATURE REVIEW}

Predicting and identifying the actions is always a complex field for research and computer practitioners and researchers

Revised Manuscript Received on September 14, 2019.

Dr.K.R.Baskaran, Professor, Department of Computer Science and Engineering, Kumaraguru College of Technology, Coimbatore, TamilNadu, India.(Email: baskaran.kr.it@kct.ac.in)

M.N.Saroja, Assistant Professor, Department of Information Technology, Kumaraguru College of Technology, Coimbatore, TamilNadu, India.(Email: saroja.mn.it@kct.ac.in) have made numerous predictions out of it. In the growing and developed IoT field, recognition of activity is of high importance. It is essential to predict the activities and assure that the developed classifier algorithms are superior in performance. By using wearable sensors, data is collected and they are pre-processed.

Several applications in pervasive computing can be considered in all kind of scenarios like medical, security and other entertainments [11]. The sensor-based activity recognition research has made remarkable progress in many disciplines [10].

Wireless technology that recognizes gesture recognition, human daily activity detection, classification, indoor location and human body monitoring, vital sign detection, imaging, and emotional recognition [15]. Increasing detection accuracy is a major challenge with increase in network traffic [16]. The Gaussian mixture modeling and Regression has built up many representations regarding motion of the human body parts [18].

\section{SYSTEM DESIGN}

In this section, HAR data is collected for giving inputs to different classifier algorithms. First, analyze the data in order to extract information. Use these data for training to build and validate a model based on the selected features through the Dictionary Learning Algorithm, Naive Bayes and J48. The Figure 1 describes the system model based on classifier algorithms.

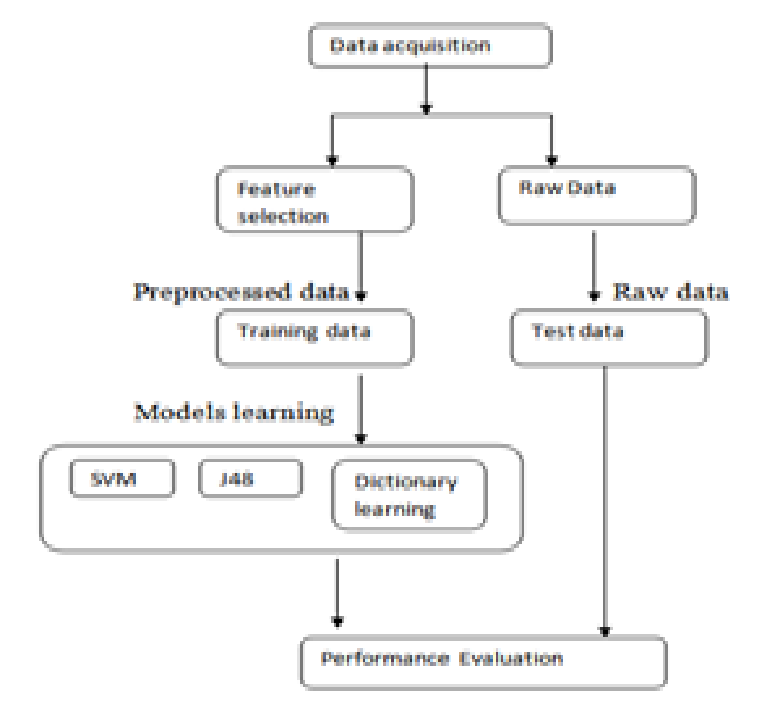

Figure 1: System Overview 


\section{A. Dictionary Learning Algorithm}

In Dictionary learning algorithm, the signal refers to the training set of data. It has two stages; offline and online stages. In offline stage, it collects the input signal from the sensors, clusters those signals and initializes the dictionary. K-SVD algorithm is widely used for learning the dictionary from the actual data size and initializes it. In online stage, the learning iteratively gets updated based on the new signal generated.

To extract the time series data, two methods are used: structural and statistical. In structural, it describes the correlation with the data. In statistical, the Fourier and Wavelet transform extract the features by quantitative characteristics of the data. It represents the flexibility and processing stages of data compared to MOD, K-SVD.

In real time, recognizing the activities is not workable. The dictionary learning algorithm helps to overcome this problem. The dictionary generated from a large training set is smaller in size and it helps to overcome the problem of computational overhead.

\section{B. J48 Algorithm}

J48 classifier is based on decision tree and is used for classification. J48 classifier creates a binary tree for classification. This decision tree built is used for building the model for classification. After the tree is developed, it will be validated on test data.

\section{Nä̈ve Bayes Classifier}

Naïve Bayes Classifier is the simplest classifier which is based on Bayes Theorem. Naive Bayes algorithm is called probabilistic classifier, which discusses the probability of any object by considering some characteristic that belong to a particular range of classes. As the Naïve Bayes algorithms makes the assumption that the occurrence of one feature is independent of other, it is called naive.

Bayes Theorem is stated as:

$$
\mathrm{P}(\mathrm{A} \mid \mathrm{B})=\mathrm{P}(\mathrm{B} \mid \mathrm{A}) \mathrm{P}(\mathrm{A}) \mathrm{P}(\mathrm{B}) \quad \rightarrow(1.1)
$$

\section{IMPLEMENTATION RESULTS}

The implementation results got by using three algorithms are presented here. First, the datasets are collected and preprocessing done for datasets like human activity recognition. The Net beans and activity recognition datasets can act as a back end and Weka tool as a front end. Finally, the accuracy and processing time are compared between Dictionary learning algorithm and J48, Support Vector Machine model. The proposed methodology will be useful in monitoring the human activities of self-dependent old aged people.

The performance of J48 classifier is shown in Table 1, whereas the performance of Naïve Bayes and Dictionary learning are shown in Table 2 and Table 3 respectively.
Table 1: Classification accuracy and processing time of J48

\begin{tabular}{|c|c|c|c|c|c|c|}
\hline $\begin{array}{c}\text { \% } \\
\text { Split }\end{array}$ & TP & FP & Precision & Recall & $\begin{array}{c}\text { Accuracy } \\
(\%)\end{array}$ & $\begin{array}{c}\text { Time } \\
\text { processing } \\
(\mathbf{s})\end{array}$ \\
\hline 30 & 0.76 & 0.056 & 0.750 & 0.760 & 75.986 & 0.47 \\
\hline 50 & 0.76 & 0.049 & 0.749 & 0.767 & 76.709 & 0.16 \\
\hline 75 & 0.774 & 0.048 & 0.772 & 0.774 & 77.371 & 0.14 \\
\hline
\end{tabular}

Table 2: Classification accuracy and processing time of Naïve Bayes

\begin{tabular}{|c|c|c|c|c|c|l|}
\hline $\begin{array}{c}\text { \% } \\
\text { Spli } \\
\mathbf{t}\end{array}$ & TP & FP & Precision & Recall & $\begin{array}{l}\text { Accur } \\
\text { acy } \\
(\%)\end{array}$ & $\begin{array}{l}\text { Time } \\
\text { processin } \\
\text { g (s) }\end{array}$ \\
\hline 30 & 0.552 & 0.068 & 0.625 & 0.552 & 55.214 & 0.10 \\
\hline 50 & 0.575 & 0.070 & 0.635 & 0.575 & 57.539 & 0.08 \\
\hline 75 & 0.063 & 0.069 & 0.642 & 0.633 & 63.283 & 0.06 \\
\hline
\end{tabular}

Table 3: Classification accuracy and processing time of Dictionary Learning

\begin{tabular}{|c|c|c|c|c|c|l|}
\hline $\begin{array}{l}\text { \% } \\
\text { Split }\end{array}$ & TP & FP & $\begin{array}{l}\text { Preci } \\
\text { sion }\end{array}$ & Recall & $\begin{array}{l}\text { Accura } \\
\text { cy (\%) }\end{array}$ & $\begin{array}{l}\text { Time } \\
\text { processing(s) }\end{array}$ \\
\hline 30 & 0.755 & 0.048 & 0.752 & 0.755 & 75.517 & 0.52 \\
\hline 50 & 0.752 & 0.047 & 0.746 & 0.752 & 75.216 & 0.28 \\
\hline 75 & 0.762 & 0.044 & 0.761 & 0.762 & 76.169 & 0.19 \\
\hline
\end{tabular}

Comparison of the performance of standard machine learning approaches is done to recognize the activities of daily living. This comparison highlights the different algorithm performances in terms of true positives, false positives, accuracy, recall, precision and specificity. $75 \%$ of the dataset is trained and the remaining is tested.

Table 4 shows the comparison results in terms of accuracy and computational time of Dictionary learning, J48 and Naive Bayes for the given dataset. The experimental results demonstrate that $\mathrm{J} 48$ and Dictionary learning algorithm gets $77.371 \%$ and $76.169 \%$ classification accuracy respectively and that of Naïve Bayes is $63.283 \%$. For the given dataset J48 algorithm gives better accuracy than the other two algorithms. 


\begin{tabular}{|c|l|l|l|l|l|l|}
\hline $\begin{array}{c}\text { Classifier } \\
\text { s }\end{array}$ & TP & FP & $\begin{array}{c}\text { Precisi } \\
\text { on }\end{array}$ & Recall & $\begin{array}{c}\text { Accur } \\
\text { acy } \\
(\%)\end{array}$ & $\begin{array}{c}\text { Time } \\
\text { proces } \\
\text { sing(s) }\end{array}$ \\
\hline $\mathrm{J} 48$ & $\begin{array}{l}0.77 \\
4\end{array}$ & 0.048 & 0.772 & 0.774 & 77.371 & 0.14 \\
\hline $\begin{array}{c}\text { Naive } \\
\text { Bayes }\end{array}$ & 0.06 & 0.069 & 0.642 & 0.633 & 63.283 & 0.06 \\
\hline $\begin{array}{c}\text { Dictionary } \\
\text { learning }\end{array}$ & $\begin{array}{l}0.76 \\
2\end{array}$ & 0.044 & 0.761 & 0.762 & 76.169 & 0.19 \\
\hline
\end{tabular}

Table 4: Performance comparison of algorithms

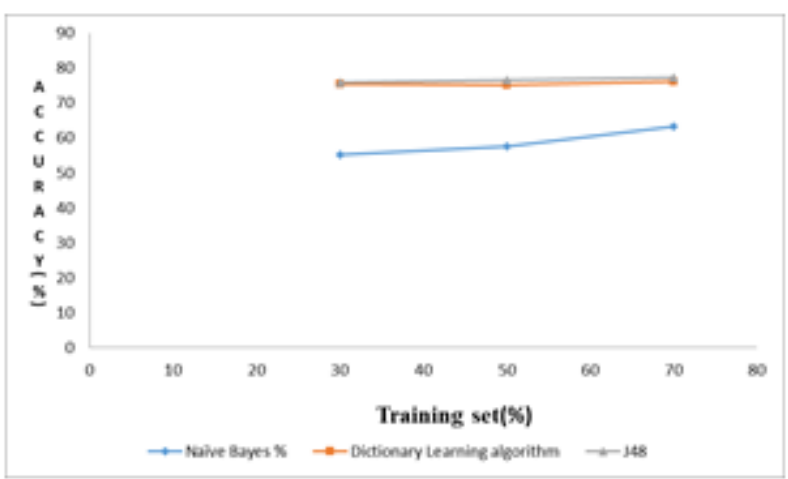

Figure 2: Classification accuracy of Different algorithms

\section{CONCLUSION}

This paper represents the activity recognition of aged people and monitors their activities through Dictionary Learning Algorithm, J48 and support vector machine algorithms. There are some drawbacks in current system like privacy as well as efficient tracking and accuracy. In future work, it is proposed to explore various models and their efficiency through many sensor data and to extract the data from different physical activities of the aged people.

\section{REFERENCES}

1. Pubali De, Amitava Chatterjee, and Anjan Rakshit (2018), 'Recognition of Human Behavior for Assisted Living Using Dictionary Learning Approach', IEEE Sensors Journal, pp. 2434-2441.

2. Uriel Martinez-Hernandez, Imran Mahmood, and Abbas A. Dehghani Sanij (2018),'Simultaneous Bayesian Recognition of Locomotion and Gait Phases With Wearable Sensors', IEEE Sensors Journal, pp. 12821290.

3. Akram Bayat, Marc Pomplun, Duc A. Tran (2014), 'A Study on Human Activity Recognition Using Accelerometer Data from Smart phones', Elsevier International Conference on Mobile Systems and Pervasive Computing, pp. 450-457.

4. Bo Tang, Jin Xu, Haibo Heand Hong Man (2017),'ADL Active Dictionary Learning for Sparse Representation', IEEE Sensors journal, pp. 2723-2729.

5. Eunju Kim, Sumi Helal, and Diane Cook (2010),'Human Activity Recognition and Pattern Discovery', IEEE Signal Processing, pp.48-53.

6. Christian Debes, Andreas Merentitis, Sergey Sukhanov, Maria Niessen, Nicolaos Frangiadakis, and Alexander Bauer (2016), 'Monitoring Activities of Daily Living in Smart Homes' ,IEEE Signal processing, pp.84-94.

7. Meisam razaviyayn, hung-wei tseng, zhi-quan luo (2014), 'Dictionary learning for sparse representation complexity and algorithms', IEEE international conference, pp.5247-5251.
8. Rong liu, ting chen, lu huang (2010),'Research on human activity recognition based on active learning' , IEEE, pp.285-290.

9. Yan Wang, Shuang Cang, Hongnian Yu1, 'A noncontactsensor surveillance system towards assisting independent living for older people', Research gate, December 2017.

10. Liming Chen, Jesse Hoey, Chris D. Nugent, Diane J. Cook, and Zhiwen Yu (2012), 'Sensor-Based Activity Recognition', IEEE Transactions On Systems, pp.790802.

11. O' scar D. Lara and Miguel A. Labrador (2013), 'A Survey on Human Activity Recognition using Wearable Sensors', IEEE Communications Surveys \& Tutorials, pp. 1192-1209.

12. Ong Chin Ann, Lau Bee Theng (2014),'Human Activity Recognition A Review', 2014 IEEE International Conference on Control System, Computing and Engineering, pp.389-393.

13. Kai-Chun Liu, Chien-Yi Yen, Li-Han Chang, Chia-Yeh Hsieh and Chia-Tai Chan (2017), 'Wearable SensorBased Activity Recognition for Housekeeping Task', IEEE sensors journal, pp.67-70.

14. Monica-Andreea Dragan and Irina Mocanu (2013),'Human Activity Recognition in Smart Environments', International Conference on Control Systems and Computer Science, pp.495-502.

15. Chao Wang, Siwen Chen, Yanwei Yang, Feng $\mathrm{Hu}$, Fugang Liu, and Jie Wu (2018), 'Literature Review on Wireless Sensing-Wi-Fi Signal-Based Recognition of Human Activities' International conference on control systems, pp.203-222.

16. Hamed Rezaie, Mona Ghassemian (2016), 'An adaptive algorithm to Improve energy efficiency in human activity recognition system' IEEE sensors journal, pp.386-395.

17. Ferhat Attal, Samer Mohammed, Mariam Dedabrishvili , Faicel Chamroukhi ,Latifa Oukhellou and Yacine Amirat (2015),' Physical human activity recognition using wearable sensors', IEEE sensors, pp. 31314-31338.

18. Barbara Bruno, Fulvia Mastrogiovanni, Antonio Sgorbissa, Tullio Vemazza and Renata Zaccaria (2016), 'Human Motion Modelling and Recognition a Computational Approach', IEEE conference on Automation on science and engineering, pp.156-161.

19. J.K. Aggarwala, Lu Xiaa (2014), 'Human Activity Recognition From 3D Data A Review', Elsevier Pattern recognition, pp.420-432. 